\title{
Multi-Vehicle Flocking: Scalability of Cooperative Control Algorithms using Pairwise Potentials
}

\author{
Yao-Li Chuang ${ }^{1,2}$, Yuan R. Huang ${ }^{3}$, Maria R. D’Orsogna ${ }^{1, \dagger}$, Andrea L. Bertozzi ${ }^{1}$
}

\begin{abstract}
In this paper, we study cooperative control algorithms using pairwise interactions, for the purpose of controlling flocks of unmanned vehicles. An important issue is the role the potential plays in the stability and possible collapse of the group as agent number increases. We model a set of interacting Dubins vehicles with fixed turning angle and speed. We perform simulations for a large number of agents and we show experimental realizations of the model on a testbed with a small number of vehicles. In both cases, critical thresholds exist between coherent, stable, and scalable flocking and dispersed or collapsing motion of the group.
\end{abstract}

\section{INTRODUCTION}

\section{A. Motivation}

Social aggregation is a remarkable aspect of animal behavior. Large numbers of individual agents interacting with each other are able to self-organize into complex yet coordinated patterns such as insect swarms, fish schools and bird flocks [1]. These systems have recently become of great interest for the mathematical [2], physical [3], [4] and biological sciences [5] with promising applications for the development and control of autonomous, multi-vehicular ensembles [6], [7]. One of the main goals of this nascent field of research is to program interactions among individuals so that desired collective behaviors may arise. The emergence of spatial patterns however, can be dramatically affected by even small parameter changes in interactions among individuals, in constituent number or speed [8]. In this paper, we formulate criteria, valid for general pairwise interactions, to ensure local group cohesion of a first order model. When interactions are controlled by a Morse potential, we investigate stability and scalability through numerical simulations and practical testbed applications, demonstrating the existence of thresholds and cutoffs for different regimes of aggregation.

\section{B. Related work and outline}

Swarming vehicular systems are often modeled as twodimensional point particles in which members may interact with one another through attractive-repulsive pairwise interactions. Specific potential choices lead self-propelled or kinematic particles to self-organize into coherent patterns [4], [9], [10], [11], [12]. More recently, swarm stabilization or collapse with increasing constituent number has been

\footnotetext{
${ }^{1}$ Dept. of Mathematics, University of California Los Angeles, Los Angeles, CA 90095 \{chuang, dorsogna, bertozzi\}emath.ucla.edu

${ }^{2}$ Dept. of Physics, Duke University, Durham, NC 27708

${ }^{3}$ Dept. of Electrical Engineering, University of California Los Angeles, Los Angeles, CA 90095 yuanh@seas.ucla.edu

$\dagger$ corresponding author
}

predicted [8]. Virtual leaders [6] and structural potential functions [13], [14] can be introduced to direct and stabilize vehicles into desired formations or to avoid obstacles. The robustness of various algorithms in the presence of noise, communication delays and other non-idealities, have been tested on several testbeds, both for single and multi-vehicular systems [15], [16]. Activities such as spatial dispersion, gradient navigation, and cluster formation have also been reported [17] as well as single-vehicle path following, stationary obstacle avoidance, and cooperative searching [18], [19].

The subject of flock cohesion for first order systems has been analyzed in detail in Refs. [9], [10], [11], where the attractive-repulsive interaction is specified and always has the unphysical feature of being unbounded for large distances. The proof that agents converge to a finite region in space depends heavily on this assumption. In the present work, on the other hand, we present a general theory applicable to any first order kinematic system subject to interactions, and find local conditions for flock cohesion. We apply our theory to the specific case of the Morse potential, which decays exponentially at large distances and represents a much more realistic description of natural and artificial swarming agents. The theory is presented in section II where we also compare our results with known properties of second order dynamic descriptions. In section III we adapt our model to a group of Dubins vehicles [20], [21] with specific attractive and repulsive interactions. We discuss stability and scalability of the system for certain parameter ranges, and we also investigate the effects of virtual leaders. Finally, in section IV, results from numerical simulations and experimental realizations of the model for small vehicle numbers are shown.

\section{THEORY}

\section{A. First order models}

We consider a general potential flow for a particle at position $\vec{r}_{i}$, at distance $r_{i}=\left|\vec{r}_{i}\right|$ from the origin, subject to dissipation $\gamma$ and to pairwise interactions $U$ :

$$
\dot{\vec{r}}_{i}=-\gamma \vec{\nabla}_{i} \sum_{j \neq i} U\left(r_{i j}\right) .
$$

Here $r_{i j} \equiv\left|\vec{r}_{i}-\vec{r}_{j}\right|$ denotes the distance between agents $i, j$. For simplicity in the remainder of this paper we will set $\gamma=1$. The potential $U$ has an attractive and repulsive part denoted by $U_{a}, U_{r}$, respectively. Then, $U^{\prime}=U_{a}^{\prime}-U_{r}^{\prime}$, with $U_{a}^{\prime}, U_{r}^{\prime} \geq 0$. The center of mass $\vec{x}=\sum_{i=1}^{N} \vec{r}_{i}$ is stationary for 
any interaction potential that depends solely on the distance between agents. Without loss of generality we let $\vec{x}=0$.

A free agent is defined as one whose distance to all other members of the swarm is greater than the repulsive length scale of the potential. In Ref. [9], free agents interact through an ad-hoc potential that, at large enough distances, is essentially a spring. This unrealistic attraction increases with distance, so that two free agents infinitely far from each other are also infinitely attracted to each other. It is not surprising then, that such free agents converge to an absorbing ball around the center of mass with finite convergence time: the crucial point in the proofs is the strongly attractive, yet unphysical, nature of the interactions at infinite distances. In particular, agents are shown to collapse inside the absorbing region, regardless of constituent number $N$ and initial condition. The radius of the absorbing ball is independent of $N$ so that the density of the final resting state diverges as $N \rightarrow \infty$.

In this paper we find the conditions on a general interaction $U$ for which this collapsing behavior can be proven locally, that is if all agents start inside a fixed set. We will later particularize this theorem to the case of a Morse potential, that has the much more realistic feature of decaying to zero as the interparticle distance becomes large. We make the following definition:

Definition 1 : Diffused state. A flock is in a diffused state if $r_{i j}>\delta \forall i \neq j$, where $\delta$ is the repulsive range such that $U^{\prime}(r)>0$ for all $r>\delta$.

Note that in order to be in a diffused state, the potential must yield only attraction outside of a certain radius. The following Lemma shows that, regardless of the specific form of the potential, a diffused state always shrinks.

Lemma 1 : Weak maximum principle. Define the flock radius as $R \equiv \sup _{i} r_{i}$. For a flock in the diffused state, $\dot{R} \leq 0$.

Proof : Let $R=r_{i}$ and define $\hat{r}_{i j} \equiv \vec{r}_{i j} / r_{i j}$. From Eq. 9 then:

$$
\begin{aligned}
\frac{{\dot{r_{i}}}^{2}}{2}=\vec{r}_{i} \cdot \dot{\vec{r}_{i}} & =-\vec{r}_{i} \cdot \sum_{j \neq i}^{N} \hat{r}_{i j} U^{\prime}\left(r_{i j}\right) \\
& =\sum_{j \neq i}^{N} \frac{\left(\vec{r}_{i} \cdot \vec{r}_{j}-r_{i}^{2}\right)}{r_{i j}} U^{\prime}\left(r_{i j}\right) \leq 0
\end{aligned}
$$

since $r_{i}^{2} \geq \vec{r}_{i} \cdot \vec{r}_{j}$ and $U^{\prime}>0$ in the diffused state. Thus $r_{i}^{2}$, and $r_{i}$, are decreasing functions and $\dot{R} \leq 0$.

A corollary to the above Lemma 1 is that the swarm size decreases even if only the outermost agents are in a diffused state. This is due to the fact that the proof only uses an estimate for the farthest agents of the swarm. We now prove a local stability limit for general interactions $U$ and find conditions for particles initially constrained to a local region of radius $R$, to evolve into a more compact ball of radius $R^{*}<R$. The proof uses a Lyapunov function discussed in [9], [10].
Theorem $1:$ Existence of bound states. Consider $N$ particles located at $\vec{r}_{i}$ with $r_{i} \leq R \forall i, 1 \leq i \leq N$. If a finite constant value $K>0$ exists such that $\max \{0 \leq r \leq 2 R\}\left|K r-U^{\prime}(r)\right|<$ $K R$, then asymptotically $r_{i} \leq R^{*}$, with $R^{*}<R$.

Proof: We choose the Lyapunov function $V_{i}=r_{i}^{2} / 2$. Its time derivative obeys the following

$$
\begin{aligned}
\dot{V}_{i} & =-\vec{r}_{i} \cdot \vec{\nabla}_{i} \sum_{j \neq i} U\left(r_{i j}\right) \\
& =-\vec{r}_{i} \cdot \sum_{j \neq i} \hat{r}_{i j} U^{\prime}\left(r_{i j}\right) \\
& \leq-K N r_{i}^{2}+r_{i}(N-1) \eta,
\end{aligned}
$$

where $\eta \equiv \max _{\{0 \leq r \leq 2 R\}}\left|K r-U^{\prime}(r)\right|$. In going from Eqn. 5 to Eqn. 6 we have added and subtracted $-K \vec{r}_{i}\left(\vec{r}_{i}-\vec{r}_{j}\right)$ in the sum and where $K>0$ is an arbitrary constant. We also used the fact that $\vec{r}_{i} \cdot \sum_{j \neq i}^{N} \vec{r}_{i j}=N r_{i}^{2}$. Also note that $r_{i j} \leq 2 R$ since by assumption $r_{i} \leq R$. Asymptotically then:

$$
r_{i} \leq \frac{N-1}{N} \frac{\eta}{K} \leq \frac{\eta}{K} \equiv R^{*},
$$

and we require $\eta<K R$ for this bound to be more stringent than the initial radius $R$.

Corollary 1:Existence of collapsed states. If Theorem 1 holds for all $R^{\prime}<R$ then as $t \rightarrow \infty$ the system will collapse with all particles converging at $R_{f}=0$.

Proof: This follows from the fact that for $r_{i} \geq \eta / K$, the Lyapunov function $\dot{V} \leq-K r_{i}^{2}=-2 K V_{i}$. The limit $R^{*}$ is thus reached in a time:

$$
t_{\max }=\max _{i}\left[\frac{1}{2 K} \ln \left(\frac{\eta^{2}}{2 K^{2} V_{i}(0)}\right)\right],
$$

where $V_{i}(0)$ is the Lyapunov function at time $t=0$. After $t_{\max }$ is reached, Theorem 1 can be applied again, and the iteration process can be repeated until the limit $R_{f}=0$ is reached. Theorem 1, applied to the parabolic potential of Ref. [9] is the global convergence theorem there shown $\square$.

Our control algorithm adopts a generalized Morse potential that decays at infinite distances, as would be expected for systems of vehicles with a limited communication range:

$$
U\left(r_{i j}\right)=-C_{a} e^{-r_{i j} / \ell_{a}}+C_{r} e^{-r_{i j} / \ell_{r}} .
$$

Here, $C_{a}, C_{r}$ represent the strength of the attractive and repulsive potentials, and $\ell_{a}, \ell_{r}$ their length scales, respectively. Define $\ell \equiv \ell_{r} / \ell_{a}, C \equiv C_{r} / C_{a}$. A sufficient condition for Theorem 1 is

$$
\left(\frac{C_{a}}{\ell_{a}} e^{-\frac{2 R}{\ell_{a}}}-\frac{C_{r}}{\ell_{r}}\right)<2 K R<2\left(\frac{C_{a}}{\ell_{a}} e^{-\frac{2 R}{\ell_{a}}}-\frac{C_{r}}{\ell_{r}}\right),
$$

which can be satisfied only if $\ell>C$ so that $R$ can be chosen as $2 R<\ell_{a} \ln (\ell / C)$. Thus, with the proper values of $\ell, C$ 


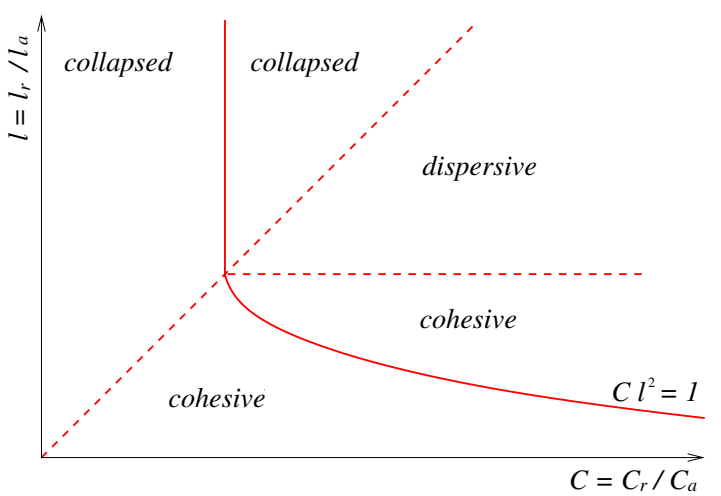

Fig. 1. Phase diagram for $N$ particles interacting according to the first order model of Eqn. 1. The region with $\ell>C$ is guaranteed to give rise to collapsed structures for any choice of $N$ if agents are initially sufficiently close to each other. In the dispersive mode particles will form an unbound system, regardless of initial conditions. In the region $\ell \leq \min \{C, 1\}$ cohesive structures form. Their shape and scaling with $N$ depends on further details of the potential and on the dimensionality of the system.

and the proper initial condition $R$, free agents subject to the non diverging Morse interactions are guaranteed to collapse to a ball of radius $R^{*}<R$. Also note that Corollary 1 holds here, since the latter condition holds for all $R^{\prime}<R$. The above condition is a sufficient but not necessary one, and other combinations of $\ell, C$ could give rise to acceptable $R, K$ values without resulting in a state where all agents collapse to a point. For other, specific choices of the potential parameters, numeric estimates can determine whether $R, K$ values exist that satisfy Theorem 1 . We can also prove that the system is dispersive for the same Morse potential in the region where $C \geq \ell \geq 1$.

Lemma 2 : Dispersion under the Morse potential. For $C \geq$ $\ell \geq 1$ of the Morse potential a bounded state at $t=0$, where $r_{k} \leq R \forall k, 1 \leq k \leq N$, will evolve into an unbounded one as $t \rightarrow \infty$.

Proof : Of the bounded particles, let $i$ be the one furthest away from the origin. We let $r_{i}=R>r_{k}$ for all $k \neq i$, so that at time $t=0, r_{i}^{2}>\vec{r}_{i} \vec{r}_{k}$. Note that to simplify the analysis we let only one particle be on the boundary, the results do not change by considering multiple particles at $R$ for $t=0$. Consider the distance between the $i$-th particle and the center of mass of the remaining $N-1$ particles. This distance is $\left|\vec{r}_{i}-\left(-\vec{r}_{i} /(N-1)\right)\right|=N r_{i} /(N-1)$ since the stationary center of mass is assumed to be fixed at the origin. The distance of the $i$-th particle from the center of mass and from the center of mass of the remaining $N-1$ particles therefore differ only by a multiplicative factor. The evolution of $\frac{1}{2} r_{i}^{2}=\vec{r}_{i} \dot{\vec{r}}_{i}$ obeys the following:

$$
\begin{aligned}
\vec{r}_{i} \dot{\vec{r}}_{i} & =\sum_{k \neq i} \frac{C_{a}}{\ell_{a}} e^{-\frac{r_{i k}}{\ell_{a}}}\left(\frac{C}{\ell} e^{r_{i k}\left(\frac{\ell-1}{\ell_{r}}\right)}-1\right) \vec{r}_{i} \hat{r}_{i k} \\
>0 &
\end{aligned}
$$

as long as $r_{i k} \vec{r}_{i} \hat{r}_{i k}=r_{i}^{2}-\vec{r}_{i} \vec{r}_{k} \geq 0$, and where we have used the fact that $C \geq \ell \geq 1$. This result indicates that $r_{i}^{2}$,

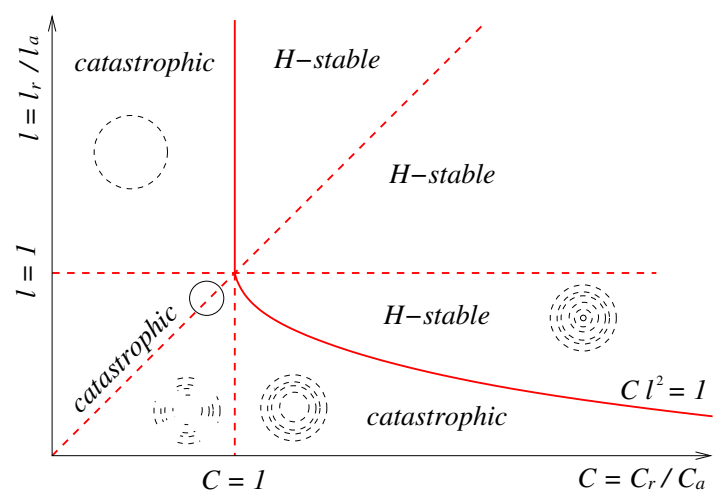

Fig. 2. Phase diagram for the second order model of Eqn. 12. Note that the system is self-propelling and asymptotically each particle will have a finite velocity, giving rise to circular or flocking structures both in the H-stable and catastrophic regimes. $\mathrm{H}$-stability permits to further characterize the details of the cohesive regime: for $C \ell^{2}<1$ cohesive structures originate that shrink in size with $N$ while patterns that are extensive with $N$ are formed in the region $C \ell^{2}>1, C \geq \ell$.

and $r_{i}$ are increasing functions in time. The $i$-th particle will thus move away from the center of mass of the other $N-1$ particles, and increase its distance from the origin as long as $r_{i} \geq r_{k}$ for all other $k$ particles. If the inequality $r_{i} \geq r_{k}$ ceases to hold, at time $t^{*}>0$ for some $k^{*}$, the outer bound of the system will be $R^{*}>R$ since $r_{i}$ has increased. Let $r_{k}^{*}>r_{i}$ at $t^{*}$ : we can then apply Lemma 2 again with $i=k^{*}$ and with the initial condition $R^{*}>R$. The system size will thus increase in an unlimited fashion.

We can adapt this result to the remainder of the $C, \ell$ phase space where $\ell \leq \min \{1, C\}$ through the following:

Corollary 2 : Cohesion under the Morse potential. For $\ell \leq$ $\min \{1, C\}$, a lower and an upper bound on the asymptotic $r_{i j}$ for all $i \neq j$ exist so that the system is cohesive.

Proof: From Eqn. 11 it is evident that for $\ell \leq \min \{1, C\}$ the distance $r_{i}$ is an increasing function of time whenever $r_{i k}<\frac{\ell_{r}}{1-\ell} \ln \left(\frac{C}{\ell}\right)$, for all $k \neq i$. This implies that any bound state of radius $R<\frac{\ell_{r}}{2(1-\ell)} \ln \left(\frac{C}{\ell}\right)$ will increase its size and will not be compacted further. On the other hand, when $r_{i k}>\frac{\ell_{r}}{1-\ell} \ln \left(\frac{C}{\ell}\right)$, for all $k \neq i$, the distance between the $i$-th particle and the center of mass of the other $N-1$ particles will decrease and particles will reside into a more compact ball. The system is thus of a cohesive type.

In this parameter region $\ell \leq \min \{1, C\}$ the potential consists of a short range repulsion and of a long range attraction. Based on the above observations, we may conclude that the swarm will be formed by particles separated by distances $r_{i k}$ such that the repulsion felt by the 'closer' particles is balanced by the attraction experienced by the 'farther' ones. The final size of the cohesive swarm will depend on the total number of constituents. In the limit of large $N$ a cohesive swarm may give rise either to an extended state, with finite density or to a collapsed one where the density is diverging. As we shall see in the analysis for the second order model, other features of the total potential, and the dimensionality of the system play a major role in determining such asymptotic swarm configurations. For 
instance, consider the following qualitative arguments for the Lyapunov function $U_{i, t o t} \equiv \sum_{j \neq i} U\left(r_{i j}\right)$. In the limit of large $N$ and finite swarm area $A$, the sums can be replaced with two-dimensional integrals so that $U_{i, t o t} \simeq$ $N A^{-1}\left(C_{r} \ell_{r}^{2}-C_{a} \ell_{a}^{2}\right)$. For $C \ell^{2}>1$ this Lyapunov function will increase with $N$ so that the collapsed limit $N \rightarrow \infty$ in a finite region of space cannot be asymptotically reached. The system thus stays cohesive but does not collapse, a possibility that may occur for $C \ell^{2} \geq 1$ where the previous arguments do not hold. Similar considerations can be found in Ref. [2]. The phase diagram for the $\{C, \ell\}$ parameters is shown in Fig. 1.

\section{B. Second order models and H-stability}

In Ref. [8] we have studied the same Morse potential in a dynamic, second order system. It will be useful to compare the results of the first and second order approaches to further characterize the Morse interaction. Our second order model reads:

$$
\dot{\vec{r}}_{i}=\vec{v}_{i}, \quad \dot{\vec{v}}_{i}=f\left(v_{i}\right) \vec{v}_{i}-\vec{\nabla}_{i} \sum_{j \neq i} U\left(r_{i j}\right) .
$$

Here, self propulsion and drag of an individual are introduced through $f$, and the potential $U$ is as above. The system is conservative if $f=0, \forall v$, and $f$ is chosen so that there exists a special value $v^{*}$ for which $f\left(v^{*}\right)=0$. As pumping and dissipation occur through $f$, it is reasonable to expect that the steady state configurations of Eqn. 12 are minimizers of the energy $U=\sum_{i \neq j} U\left(r_{i j}\right)$ and zeroes of $f$.

Drawing on analogies with statistical ensembles [22], in Ref. [8] we show that an important indicator of the expected morphology is the $H$-stability of the interaction potential $U$. A system is said to be $\mathrm{H}$-stable if the energy per particle is bounded from below as the number of particles goes to infinity. Mathematically, a system is H-stable if a constant $B>0$ exists such that:

$$
\lim _{N \rightarrow \infty} \sum_{j \neq i}^{N} U\left(r_{i j}\right) \geq-B N
$$

In the limit $N \rightarrow \infty$, H-stable interactions result in particles either occupying the entire space at their disposal in a gaslike manner and with zero density, or keeping interparticle distances fixed, so that the density remains constant. In the language of the purely dissipative model of Eqn.1, $\mathrm{H}$-stable interactions correspond to dispersed or cohesive agent behavior. In the latter case, a finite nearest-neighbor distance emerges as $N \rightarrow \infty$. Non H-stable potentials, on the other hand, are called 'catastrophic' as they typically result in systems that collapse to a localized region in space with diverging density in the $N \rightarrow \infty$ limit. For finite $N$ catastrophic potentials give rise to cohesive motions of agent groups. As $N \rightarrow \infty$ nearest-neighbor distances become vanishingly small, and the group eventually collapses. The potentials analyzed in Ref. [9] are all examples of catastrophic potentials for the dynamic system.
We compare the results for the first order model of Eqn. 1 to the the phase diagram arising from the second order model of Eqn. 12 in Fig. 2. The region $\ell>C$ with $\ell<1$ is classified as catastrophic in Ref. [8], with particles converging towards their center of mass and becoming denser as $N \rightarrow \infty$. This is consistent with the results proven here that $N$ particles initially in a ball of radius $R$ get 'squeezed' into a tighter one. On the other hand, the region $\ell>C$, with $\ell>1$ is classified as stable in Ref. [8], with no possible squeezing effects in the long time limit. This can be understood as follows. In the region $\ell>C, \ell>1$, the pairwise potential has a positive, local minimum for $r_{i j}=0$ and a barrier at $r_{i j}=r_{\max }>\ell_{a} \ln (\ell / C)$, before decaying to zero as $r_{i j} \rightarrow \infty$. The first order system (1) is purely dissipative and there are no fluctuations in the total energy, which can only decrease in time. For second order systems of the type described in Eqn. 12 however, even if the local energy minimum is reached, with all particles simultaneously at $r_{i j}=0$, fluctuations due to exchange with the environment as imposed by $f$, can eventually drive the system away, towards the dispersed, global energy minimum at $r_{i j} \rightarrow \infty$.

\section{TESTBED ADAPTATION}

The models described in Eqns. 1 and 12 cannot be directly applied to the specific platform of autonomous vehicles we are equipped with, due to mechanical constraints that limit speed and turning radii capabilities. The real vehicles we use are described in Ref. [16] and consist of Dubins micro-cars with fixed speed and fixed left and right turning radii. The first constraint implies our dynamical system must be described as first order. The only independent variable denoting agent $i$ is its heading angle with respect to a fixed orientation we define as $\theta_{i}$. The Dubins vehicles interact with each other by means of the Morse potential of Eqn. 9 with variable parameters $C_{a}, C_{r}, \ell_{a}, \ell_{r}$. Due to the fixed turning radii, the interactions cannot directly control $\theta_{i}$ and an appropriate control algorithm must be devised. For each vehicle then, we measure the angle $\gamma_{i}$ between vehicle heading and the total force $\vec{F}_{i}$ it experiences, as given by the right hand side of Eqn. 1 and as shown in Fig. 3. Vehicle $i$ then changes direction only if $\left|\gamma_{i}\right|>\Gamma$, where $\Gamma$ is an angular threshold $0 \leq \Gamma \leq \pi$. The equations of motion are as follows:

$$
\begin{aligned}
& \dot{x}_{i}=\alpha \cos \theta_{i} \quad \dot{y}_{i}=\alpha \sin \theta_{i}, \\
& \dot{\theta}_{i}=\left\{\begin{array}{cll}
\frac{\alpha}{R_{L}} & \text { if } \gamma_{i}>\Gamma \quad \text { (left turn) } \\
-\frac{\alpha}{R_{R}} & \text { if } \gamma_{i}<-\Gamma \quad \text { (right turn), } \\
\frac{\alpha}{R_{S}} & \text { otherwise. }
\end{array}\right.
\end{aligned}
$$

Here, $\alpha$ is the speed of the vehicle, and $R_{L}, R_{R}$ are the left and right turning radii, respectively. $R_{S}$ is the deviation radius. In the ideal case $R_{L}=R_{R}$ and $R_{S}=\infty$, so that vehicle direction is unaffected for $\left|\gamma_{i}\right|<\Gamma$. Because of alignment asymmetries, in general $R_{L} \neq R_{R}$ and $R_{S}$ is a large but finite number. Vehicular motion proceeds along 


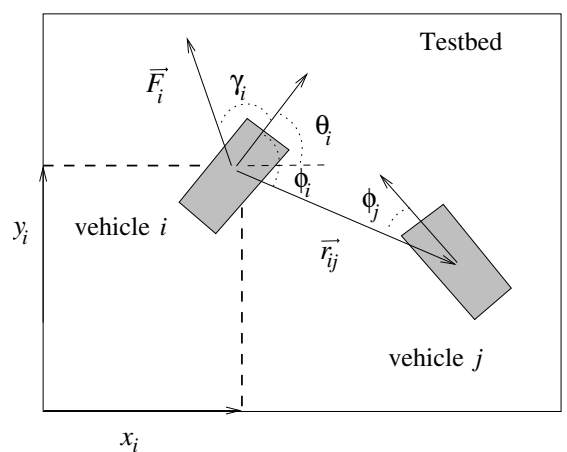

Fig. 3. Definition of variables for vehicle $i$ : The heading is denoted by $\theta_{i}$, the angle between its direction of motion and the $x$ axis of the testbed. $\vec{F}_{i}$ is the interaction force it experiences due to all other vehicles. This direction defines an angle $\gamma_{i}$ with the heading direction. Vehicle $i$ is at a distance $\vec{r}_{i j}$ from vehicle $j$ and the angles $\phi_{i}$ and $\phi_{j}$ here shown are used in the collision avoidance scheme described in the text. The origin of the reference coordinate system is fixed at the left-lower corner of the testbed. All vehicular angles, $\gamma_{i}, \theta_{i}, \phi_{i}$, are defined in $[\pi,-\pi)$.

the direction specified by the heading parameter $\theta_{i}$ until the turning commands $\dot{\theta}_{i}$ are given.

A crucial point is that the interaction potential in Eqn. 9 is soft-core and does not prevent vehicles from colliding. In fact, even hard-core potentials cannot avoid collisions due to communication delays, errors in position information, and the finite turning radii of the vehicles. The repulsive range may be increased to initiate turning at larger inter-vehicle distances. This however, would significantly affect pattern formation and the emergence of cooperative aggregates would be unlikely. Instead, we add an additional collision avoidance algorithm to address short range interactions. We use a 'wait and go' scheme for vehicles closer than a cutoff distance $r_{c}$. For vehicles $i, j$ at distance $\vec{r}_{i j}$ such that $r_{i j}<r_{c}$, we define the angles $\phi_{i}, \phi_{j}$ between their main axis and $\vec{r}_{i j}$, as shown in Fig. 3. If $\phi_{i}<\phi_{j}$ vehicle $i$ will pause while vehicle $j$ veers away, until $r_{i j}>r_{c}$. The cutoff distance $r_{c}$ in the control algorithm acts as an effective hardcore potential. If $\phi_{i}=\phi_{j}$ any one of the vehicles (in our simulations the one with a higher labeling index) will pause and let the other proceed. When $\phi_{i}, \phi_{j} \simeq 0$ the 'wait and go' scheme cannot avoid collision as shown in Fig. 4, and an alternate algorithm is invoked. For vehicles $i$ and $j$ we define the angle $\Omega_{i j}$ between $\vec{r}_{i j}$ and the segment joining their opposite front edges measured from $\max \left\{\phi_{i}, \phi_{j}\right\}$ as shown in Fig. 4. If $\max \left\{\phi_{i}, \phi_{j}\right\}<\Omega$, where $\Omega$ is an angular threshold $0 \leq \Omega \leq \pi / 2$, then the vehicle closer to the center of the testbed is veered towards the center and the other in the opposite direction.

\section{EXPERIMENTAL RESULtS}

In this section we study the behavior and performance scaling of a set of Dubins vehicles controlled by the first order laws based on the model in the previous section. We consider both testbed implementation and numerical simulations for small and large numbers of vehicles, respectively. The computer model is validated against the testbed in the case of a few vehicles. It is also possible to incorporate the presence of

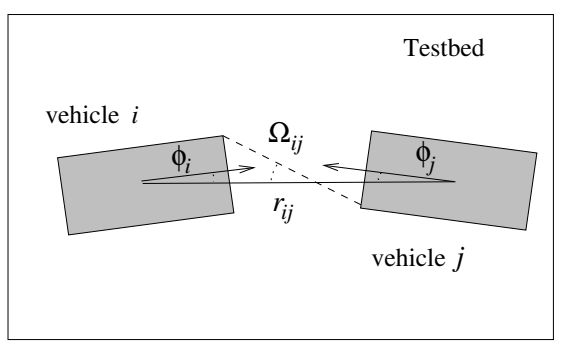

Fig. 4. Collision avoidance failure: The angles $\phi_{i}$ and $\phi_{j}$ are too small and vehicles $i$ and $j$ collide even if one of them should pause. An additional algorithm is required to steer the vehicles away from each other and is described in the text. It relies on the angle $\Omega_{i j}$ here depicted.

many virtual vehicles in practical testbed applications and study the effects of larger vehicle numbers on the actual ones.

\section{A. Testbed Simulations}

The testbed has three working vehicles. A virtual leader moves around an ellipse with semimajor axis approximately 15 times the vehicle length. There is some variability in vehicle speed. To address this issue, the position of the leader is checked against the distance to the closest vehicle. If the distance becomes larger than a certain threshold $d_{t}$, the leader will pause; otherwise, it will move at its intrinsic speed, We select our parameters as follows: $\ell_{r}=5.7 \mathrm{~cm}, \ell_{a}=95.2 \mathrm{~cm}$, $C_{a}=10^{4} \mathrm{erg}$ and $C_{r}=6 \cdot 10^{3} \mathrm{erg}$. so that $C=1.67$ and $\ell=$ 0.06 . Note that these parameters correspond to a potential in the 'catastrophic regime' of Ref. [8]. For potential parameters in the H-stable regime we have not been able to realize stable configurations of vehicular aggregation due, in part, to the constant speed of the vehicles. The leader interacts with the vehicles according to the same Morse potential used for vehicle-vehicle interaction. When leading more than one vehicle, the leader's contribution to the potential is increased 1.1 times and 2.1 times the vehicular potential for the twovehicle and the three-vehicle experiments, respectively.

1) One vehicle follows a leader: The parameters mentioned above provide short-range repulsion and long-range attraction resulting in an equilibrium separation. Figure 5 shows results for $d_{t}$ near the equilibrium $r_{e q}$, calculated to be $r_{e q}=20.2 \mathrm{~cm}$. Running tests with $d_{t}=20.5 \mathrm{~cm}$, $d_{t}=20.2 \mathrm{~cm}$, and $d_{t}=20.0 \mathrm{~cm}$, we note that leaderfollowing becomes ineffective for $d_{t}$ below $r_{e q}$.

2) Two vehicles follow a leader: The vehicles are found to alternate between a snake-like competing behavior as shown in Fig. 6-top and a stable gliding behavior as shown in Fig. 6middle. The stable behavior emerges when one vehicle trails the other and they form a rather flat triangle with the leader that glides around the ellipse as shown in Fig. 6-bottom.

3) Three vehicles follow a leader: The vehicles still alternate between competing and gliding behaviors as in the two-vehicle case as shown in Fig.7-top. When stable motion emerges, the vehicles and the leader form a stretched quadrilateral that glides around the ellipse as shown in Figs. 7-middle and bottom. We note that fragmentation can 

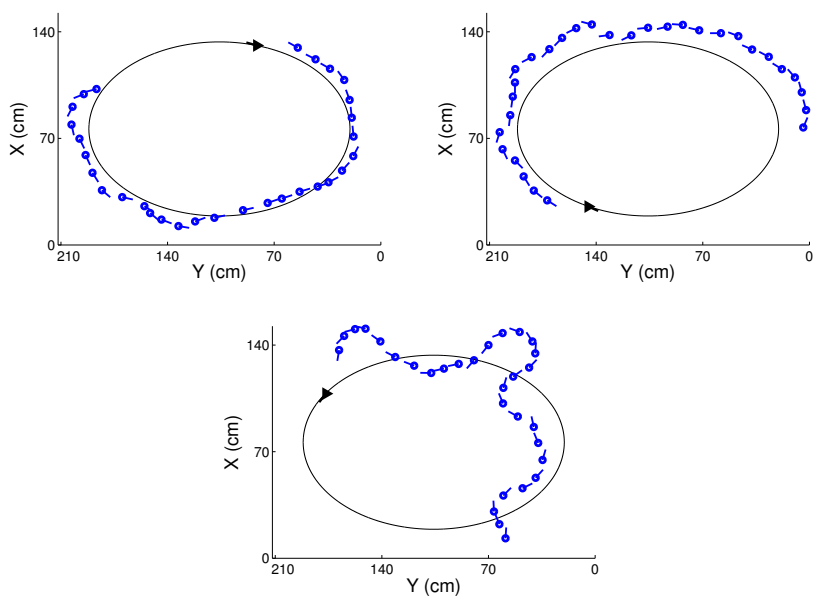

Fig. 5. Vehicular motion: These panels show fragments of the vehicle's trajectory when it tries to follow a virtual leader along an elliptical path. The vehicle is unstable when $d_{t}$ is decreased below $r_{e q}=20.2 \mathrm{~cm}$. Top left: $d_{t}=20.5 \mathrm{~cm}$; Top right: $d_{t}=20.2 \mathrm{~cm}$; Bottom: $d_{t}=20.0 \mathrm{~cm}$.
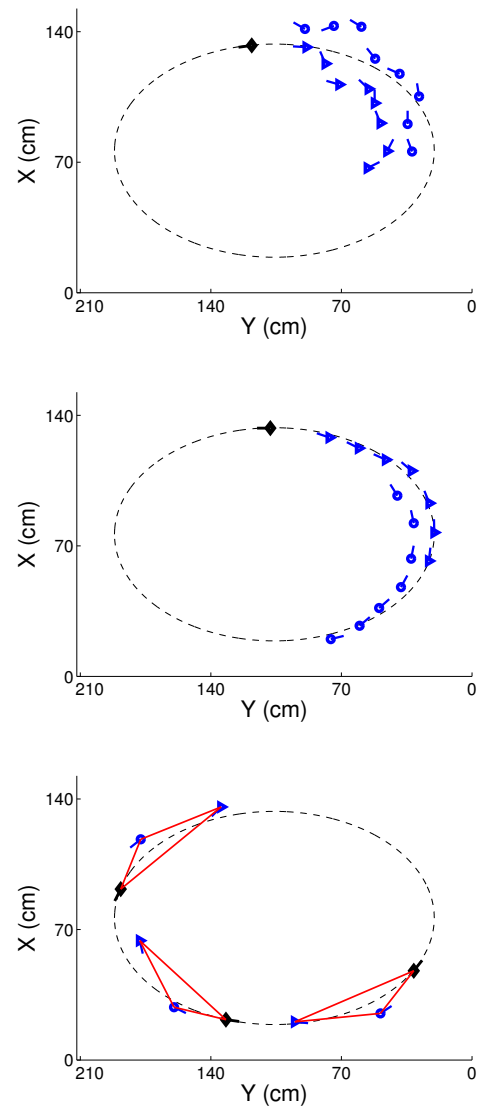

Fig. 6. Two vehicles try to follow a virtual leader along an elliptical path. Top: Two vehicles exhibit snake-like motion as they compete for the optimal spot behind the virtual leader; middle and bottom: The vehicles' motion becomes stable when one trails the other, and they form a flat triangle with the leader, which glides along the path.
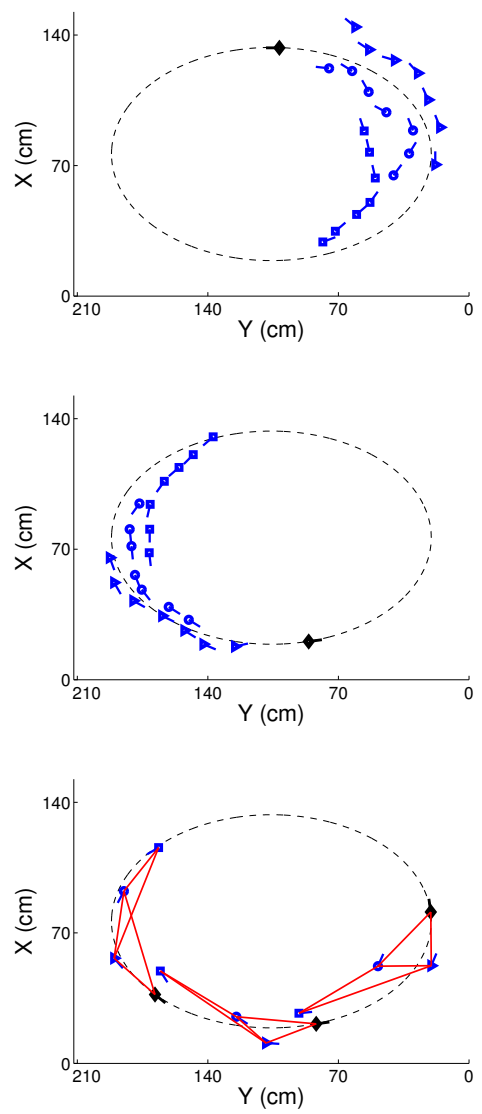

Fig. 7. Three vehicles try to follow a virtual leader along an elliptical path. Top: Vehicles exhibit snake-like motion when they level with each other; Middle: The formation becomes stable when one trails another. Bottom: The vehicles and the leader form a stretched quadrilateral that glides along the path.

sometimes occur due to the stretched formation, as the attraction between the two slower vehicles overwhelms the long-range attraction from the leader.

To reduce such occurrences, we can enhance the leader attraction by increasing its weight. Also, both group cohesion and stabilization of the above examples can be realized by imposing rigid formations for the vehicle group as in Ref. [6]. Note, however, that in the absence of a rigid structure, even though the vehicles shift position with respect to each other, they are able to maintain a coherent group as they follow the leader around the track.

\section{B. Computer Simulations}

Computer simulations provide a powerful tool to study scalability and statistical issues for large numbers of vehicles. Figure 8 shows two distinct formations observed in computer simulations of 100 vehicles. Aggregates similar to the vortex shown in the left-hand panel of Fig. 8 are seen for weak or non-existent leaders. For strong, effective leaders, vehicles align and follow, as shown in the right hand panel. For the second-order model of Eqn. 12 as specified in Ref. [8] it is shown that as the number of agents increase, collapse, stability or dispersion of the agents depend on the parameters 
of the potential. It is interesting to investigate how these results compare to the first-order model of Eqns.14 and 15. In particular, in Ref.[8] it is shown that for a range of parameter values defined by $C$ and $\ell$ coherent behavior is expected. In Fig. 9 we show the steady state formation radius as a function of vehicle number in the catastrophic regime, where coherent structures are expected to collapse as the number of constituents increases. In the present model, the size of a catastrophic flock remains steady as vehicle number increases, consistent with an increasing vehicle density. On the other hand, for parameter values in the H-stable regime, where aggregation is extensive in large number limit, the flock size expands with increasing vehicle number. Repulsion is more accentuated in the $\mathrm{H}$-stable regime: for parameters that are close to the stable-catastrophic threshold flocking is still possible, but as the parameters are chosen further and further away into the H-stable regime, cooperative flocks no longer occur and vehicle groups loose coherence. Figure 10 shows that the critical $\ell_{r}$, beyond which the flock disintegrates is located deeper into the $\mathrm{H}$-stable regime as the number of vehicles increases.

\section{CONCLUSIONS}

We consider a well-known first order gradient flow model for robot interactions in a swarm. We prove new results on cohesion and collapse for a general class of potentials. In particular, we find conditions under which the system is guaranteed to converge inside a ball of fixed radius, provided it started from a ball of pre-defined larger radius. These radii are independent of number of agents and result in a state in which swarm density goes to infinity as vehicle number increases. Such scaling results are very important in designing large agent swarming algorithms. We adapt the model to a system of Dubins vehicles and consider both testbed and numerical simulations for the swarm. We include a virtual leader which allows for continued motion of the swarm in a confined geometry. For small numbers of agents, the testbed verifies some simple facts about stability of the algorithm under certain parameters of the virtual leader potential. For large numbers of agents we show in computer simulations how the size of the swarm scales as the agent number increases. In our model, as the number of agents grows, the swarm is able to maintain its cohesion using potentials with parameters that would lead to instability at smaller numbers.

\section{ACKNOWLEDGMENTS}

This research was supported by ONR grant N000140610059 and ARO grants W911NF-05-1-0112 and 50 363-MA-MUR.

\section{REFERENCES}

[1] S. Camazine, J. L. Deneubourg, N. R. Franks, J. Sneyd, G. Theraulaz, and E. Bonabeau, Self organization in biological systems, Princeton University Press, Princeton, NJ, 2003.

[2] A. Mogilner, L. Edelstein-Keshet, L. Bent and A. Spiros, "Mutual interactions, potentials, and individual distance in a social aggregation", J. Math. Biol. Vol. 47, pp. 353-389, 2003.
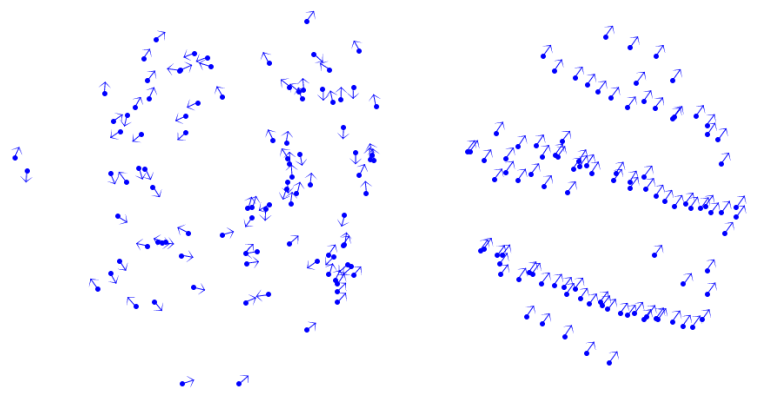

Fig. 8. Vehicular formations in the presence of a leader: The formation to the left occurs when the vehicles fall out of the leader's path and selfaggregate into a vortex-like formation. The formation to the right occurs when the vehicles successfully follow the leader.

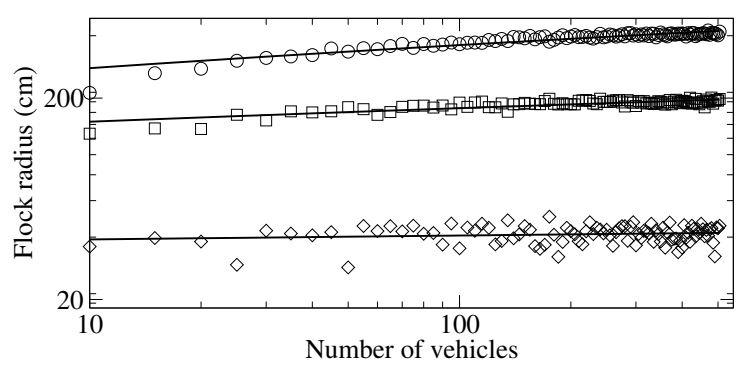

Fig. 9. Scaling in the H-stable and catastrophic regimes. The potential parameters are set at $\ell_{a}=95.2 \mathrm{~cm}, C_{a}=10^{4} \mathrm{erg}$ and $C_{r}=6 \cdot 10^{3} \mathrm{erg}$. With these parameter choices, H-stability is guaranteed for $\ell_{r}>73.5 \mathrm{~cm}$. In the top curve $\ell_{r}=76.2 \mathrm{~cm}$, in the middle one, $\ell_{r}=69.0 \mathrm{~cm}$, just below the transition threshold. The bottom curve, for which $\ell_{r}=35.7 \mathrm{~cm}$, falls deeply into the catastrophic regime. Straight lines are power law fits with powers $10^{-1}, 10^{-2}$ for the top and middle set. Within fitting errors, the catastrophic curve defines a constant flocking radius.

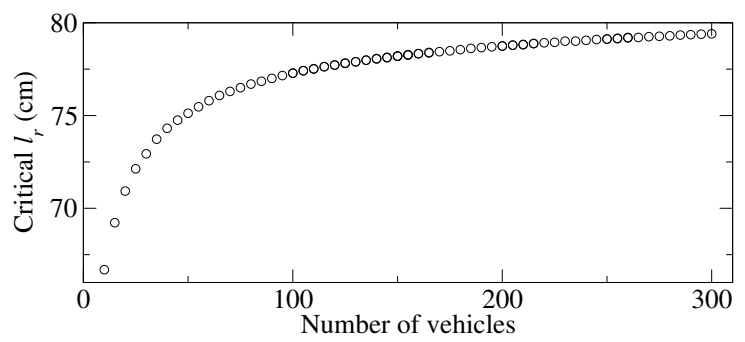

Fig. 10. Critical $\ell_{r}$ versus vehicle number. The data points indicate the $\ell_{r}$ threshold beyond which the cooperative flock disintegrates. $C_{r}, C_{a}, \ell_{a}$ are the same as in Fig. 9

[3] T. Vicsek, A. Czirok, E.B. Jacob, I. Cohen, and O. Schochet, "Novel type of phase transitions in a system of self-driven particles", Phys. Rev. Lett. Vol. 75, pp. 1226-1229, 1995.

[4] H. Levine, W. J. Rappel, and I. Cohen "Self-organization in systems of self-propelled particles", Phys. Rev. E, Vol. 63, pp. 017101, 2000.

[5] I. D. Couzin, J. Krause, R. James, G. D. Ruxton, and N. R. Franks, "Collective memory and spatial sorting in animal groups", J. Theor. Biol., Vol. 218, pp. 1-11, 2002.

[6] N. E. Leonard and E. Fiorelli, "Virtual leaders, artificial potentials, and coordinated control of groups", in Proc. Conf. Decision Contr., Orlando, FL, pp. 2968-2973, 2001.

[7] A. Jadbabaie, J. Lin, and A. S. Morse, "Coordination of groups of mobile autonomous agents using nearest neighbor rules", IEEE Trans. Autom. Contr., Vol. 48, pp. 988-1001, 2003.

[8] M. R. D’Orsogna, Y. L. Chuang, A. L. Bertozzi and L. Chayes, "Self- 
propelled particles with soft-core interactions: patterns, stability, and collapse", Phys. Rev. Lett., Vol. 96, 104302, 2006.

[9] V. Gazi and K. Passino, "Stability analysis of swarms", in IEEE Trans. Autom. Contr. Vol. 48, pp. 692-697, 2003.

[10] V. Gazi and K. Passino, "A class of attractions/repulsion functions for stable swarm aggregations", in Proc. Conf. Decision Contr., Las Vegas, NV, pp. 2842-2847, 2002.

[11] V. Gazi and K. Passino, "Stability analysis of social foraging swarms: combined effects of attractant/repellent profiles", in Prof. Conf. Decision Contr., Las Vegas, NV, pp. 2848-2853, 2002.

[12] N. Shimoyama, K. Sugawara, T. Mizuguchi, Y. Hayakawa and M. Sano, "Collective motion in a system of motile elements", Phys. Rev. Lett., Vol. 76, 3870-3873, 1996

[13] R. Olfati-Saber and R. M. Murray, "Distributed cooperative control of multiple vehicle formations using structural potential functions", in IFAC World Congress, Barcelona, Spain, 2002. http://thayer.dartmouth.edu/ olfati/papers/ifac02_ros_rmm.pdf

[14] R. Olfati-Saber, "Flocking for multi-agent dynamic systems: algorithms and theory", IEEE Trans. on Autom. Contr., Vol. 51, 2006, to appear.

[15] L. Cremean, W. Dunbar, D. V. Gogh, J. Kickey, E. Klavins, J. Meltzer, R. M. Murray, "The Caltech Multi-Vehicle Wireless Testbed", in Proc. Conf. Decision Contr., Las Vegas, NV, pp.86-88, 2002; Z. Jin, S. Waydo, E. B. Wildanger, M. Lammers, H. Scholze, P. Foley, D. Held, and R. M. Murray, "MVWT-II: The second generation Caltech MultiVehicle Wireless Testbed", in Proc. Amer. Contr. Conf., Boston, MA, pp. 5321-5326, 2004.

[16] C. H. Hsieh, Y. L. Chuang, Y. Huang, K. K. Leung, A. L. Bertozzi, and E. Frazzoli, "An economical micro-car testbed for validation of cooperative control strategies", in Proc. Amer. Contr. Conf., Minneapolis, MN, pp. 1446-1451, 2006.

[17] J. McLurkin, MIT Computer Science and Artificial Intelligence Laboratory, http://people.csail.mit.edu/jamesm/

[18] D. J. Lee and M. W. Spong, "Stable Flocking of Inertial Agents on Balanced Communication Graphs", in Amer. Contr. Conf., Minneapolis, MN, pp. 2136-2141, 2006.

[19] B. Q. Nguyen, Y. L. Chuang, D. Tung, C. Hsieh, Z. Jin, L. Shi, D. Marthaler, A. L. Bertozzi, and R. M. Murray, "Virtual attractiverepulsive potentials for cooperative control of second order dynamic vehicles on the Caltech MVWT", in Proc. Amer. Contr. Conf., Portland, OR, pp. 1084-1089, 2005.

[20] L. E. Dubins, "On curves of minimal length with a constraint on average curvature and with prescribed initial and terminal positions and tangents", Amer. J. Math., Vol. 79, pp. 497-516, 1957.

[21] A. M. Shkel and V. J. Lumelsky, "Classification of the Dubins set", Robotics and Autonomous Systems, Vol. 34, pp. 179-202, 2001.

[22] D. Ruelle, Statistical Mechanics, rigorous results, W. A. Benjamin Inc, New York, NY, 1969. 